\title{
Mehr Materialabtrag bei reduziertem Eigenabrieb
}

\author{
Für das Schleifen und Polieren von Werkstücken aus Metall, speziell von Druckgussteilen, \\ kommen neue, stark schleifende Kunststoff-Schleifkörper zum Einsatz. Durch ihre hohe Dichte
}

und scharfen Kanten der eingebetteten Kristalle verkürzen sie die Bearbeitungszeit beim

Gleitschleifen deutlich. Gleichzeitig erzielen sie einen geringeren Eigenabrieb.

$\mathrm{D}$ ie neuen HDC (High Density Cut)Schleifkörper für das Gleitschleifen bestehen aus Zirkonsilikat-Kristallen, die in ein Kunststoff-Substrat eingebettet sind, und vereinen so die Vorteile der Kunststoff-Schleifkörper mit der höheren Dichte des Werkstoffes Keramik. So erzielen sie einen Materialabtrag, der mit dem stark schleifender Keramik-Schleifkörper vergleichbar oder auch besser ist, dennoch ist der Eigenabrieb gering. Außerdem erzeugen sie ein sehr gutes Oberflächenfinish.

Die von Walther Trowal entwickelten Schleifkörper (Trowalplast HDC) eignen sich für Anwendungen, für die bisher mit Keramikkörpern gearbeitet wurde, um Material intensiv abzutragen. Wegen der Splitterneigung oder des zu starken Eigenabriebes von Keramik sind Anwender allerdings auf Kunststoff umgestiegen. Damit erreichen sie gute Ergebnisse, jedoch nur mit längeren Bearbeitungszeiten. Die

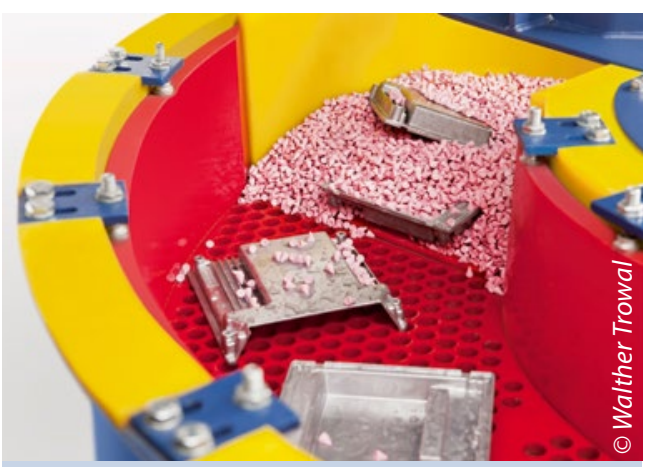

Die acht Millimeter großen Schleifkörper sind durch ihre Farbe auf den Werkstücken leicht zu erkennen neuen Schleifkörper sollen diese Lücke schließen.

Eine typische Anwendung für die neuen Verfahrensmittel sind Druckgussteile, bei denen bisher stark schleifende Kunststoffschleifkörper verwendet werden. Die HDC-Körper erlauben einen hohen Nutzen in allen Gleitschleifanlagen: Das gilt für Fliehkraftanlagen, in denen Keramikkörper nur eine begrenzte Lebensdauer haben, ebenso wie für Durchlaufanlagen, deren Durchlaufzeit nur in vergleichsweise engen Grenzen variiert werden kann. In diesen Anlagen erzielen HDC-Körper bei der vorgegebenen Durchlaufzeit deutlich bessere Ergebnisse.

\section{Hohe Schleifleistung}

Aufgrund des hohen Gewichtes und der scharfen Kanten der eingebetteten Kristalle haben die neuen Schleifkörper ähnlich gute Schleifeigenschaften wie Keramik-Schleifkörper.

Neben dem Werkstoff spielt auch die Form eine wichtige Rolle. Während keramische Körper in ihrer Formgebung begrenzt sind, können die HDC-Schleifkörper in allen Ausprägungen hergestellt werden, die von Kunststoff-Körpern bekannt ist. So kommen Schleifkörper zum Einsatz, die optimal an unterschiedliche Werkstücktypen angepasst sind.

Als erstes Produkt stellt Walther Trowal kegelförmige Schleifkörper mit einer Größe von acht Millimeter her. Sie sind die kleinsten, die auf dem Markt erhältlich sind, und eignen sich besonders für Werkstücke, die viele Innenecken aufweisen.

Im praktischen Betrieb bietet auch die Farbe Vorteile: Im Gegensatz zu den meist grauen Keramikkörpern sind die HDC-Körper rosa. So sind Schleifkörper, die im Werkstück festklemmen, leichter zu erkennen.

\section{Geringerer Eigenabrieb}

Während bisher ein hoher Materialabtrag immer mit hohem Abrieb der Schleifmittel einherging, ist das bei den HDC-Schleifkörpern anders: Obwohl sie viel Material abtragen, ist der Eigenabrieb gering. Im Vergleich mit anderen stark schleifenden Schleifkörpern weisen die HDC-Körper einen deutlich niedrigeren Abrieb auf.

\section{Frei von Silizium und Aluminium}

Für HDC verwendet Walther Trowal Zirkonsilikat. Das hat neben der Scharfkantigkeit der Kristalle den Vorteil, dass es weder Aluminiumoxid noch Siliziumdioxid enthält. So kann es für Werkstücke verwendet werden, die mit diesen Stoffen nicht in Berührung kommen dürfen. Die HDC-Schleifkörper ergänzen damit die Palette von stark und schwach schleifenden, aluminiumoxid-freien Schleifkörpern. I

\section{Kontakt:}

Klaus Peter Neidhardt,

Walther Trowal GmbH \& Co. KG, Haan, Tel. 02129571209 ,

kp.neidhardt@walther-trowal.de,www.walther-trowal.de 